\title{
Calculation and Analysis of Aircraft Infrared Detection Probability under the Single Background
}

\author{
Ma Yunpeng ${ }^{1, a}$, Zhang $\mathrm{Pei}^{2, \mathrm{~b}}$ \\ ${ }^{1}$ School of Aeronautic Science and Engineering, Beihang University, Beijing, 100191, China \\ ${ }^{2}$ School of Aeronautic Science and Engineering, Beihang University, Beijing, 100191, China \\ amyp@buaa.edu.cn, bclytze9064@163.com
}

Keywords: Detection Probability; Target Radiance; Microsoft Visual C++ 6.0

\begin{abstract}
Detection probability of aircraft infrared characteristic signal has an irreplaceable role on finding target. This paper mainly researches on aircraft infrared detection probability under the single background. First, target infrared radiation model is established, and then a weighted calculation method of aircraft critical components infrared radiation intensity is designed according to the aircraft and missile space position relations. Second, calculate the atmospheric transmittance, solar radiation intensity and atmospheric radiation intensity using LOWTRAN7 software. Third, computing platform of aircraft infrared signal detection probability is established based on Microsoft Visual C++ 6.0. The platform can calculate the detection probability of the plane accurately. Finally, the influence of location and distance aircraft relative to detector on detection probability is analyzed.
\end{abstract}

\section{Introduction}

Timely, quickly and accurately grasping the enemy forces, equipment deployment and maneuver situation on the battlefield, plays a vital role on grasping the battlefield initiative and making an accurate decision for the battlefield commander. As an important part of the battlefield reconnaissance system, target detection and location system has become a research hotspot in the field of battlefield reconnaissance.

Infrared detection technology plays an important role in investigation technology, and it is of great significance for the detection probability of IR detector to judge whether the detector can detect the target is of great importance. Detection probability is related to target radiance, background radiance, atmospheric transmittance, the distance between the detector and target, optical system parameters, etc. This paper puts forward a kind of estimation method of detection probability for the aircraft with conventional layout under the single background. The target radiance model and detection probability calculation model based on Microsoft Visual C++ 6.0 is established.

\section{Infrared radiation model}

The infrared radiation optical seeker can "see" is divided into two parts: target infrared radiation and background infrared radiation. Target infrared radiation depends on the target surface temperature, emissivity, atmospheric absorption, target geometry, detector characteristics and so on. Aircraft parameters involved in this article take F22 as reference. This paper assume that the plane fly steady and horizontally in the process of detecting. Background infrared radiation only consider the single background of sunny sky. Detailed target infrared radiation model is described as follows.

Target radiation includes own radiation, atmospheric path transmission radiation and the background radiation by reflection, and the background radiation is mainly composed of solar radiation and sky radiation.

In the wavelength of $\lambda_{1} \sim \lambda_{2}$, target infrared radiation can be expressed as Eq. 1 and Eq. 2.

$$
L_{\text {sensor }}=\int_{\lambda_{1}}^{\lambda_{2}} \tau_{\text {opt }}(\lambda)\left[L_{\text {path } \lambda}+\tau_{\text {atm }}(\lambda)\left(L_{\text {thermal } \lambda}+L_{\text {back } \lambda}\right)\right] d \lambda
$$


$L_{\text {back } \lambda}=\rho\left(L_{\text {sun } \lambda}+L_{\text {sky } \lambda}\right)$

$L_{\text {path } \lambda}$ is atmospheric path spectrum radiance. $L_{\text {thermal } \lambda}$ is target emission spectrum radiance. $L_{b a c k \lambda}$ is background spectrum radiance by the target reflection, and it is divided into solar spectrum radiance by the target reflection and sky spectrum radiance by the target reflection. $\rho$ is surface reflectivity of the target. $\tau_{\text {atm }}(\lambda)$ is the spectral transmittance of atmosphere. $\tau_{\text {opt }}(\lambda)$ is the spectral transmittance of optical system.

Target radiance model. According to the Planck's formula, blackbody spectrum radiance $M_{\lambda}$ (spectral radiant exitance) can be expressed as Eq. 3.

$$
M_{\lambda}=\frac{c_{1}}{\pi} \lambda^{-5} \frac{1}{e^{c_{2} / \lambda T}-1}
$$

$\lambda$ is the wavelength of radiant electromagnetic wave. $T$ is the thermodynamic temperature of blackbody. $c_{1}$ is the first radiation constant. $c_{2}$ is the second radiation constant.

Blackbody radiance which wavelength ranges from $\lambda_{1}$ to $\lambda_{2}$, can be expressed as Eq. 4 .

$$
L=\frac{\varepsilon_{1}}{\pi} \int_{\lambda_{1}}^{\lambda_{2}} M_{\lambda} d \lambda=M_{\lambda_{1}-\lambda_{2}}
$$

$\varepsilon_{1}$ is skin emissivity. $\int_{\lambda_{1}}^{\lambda_{2}} M_{\lambda} d \lambda=M_{\lambda_{1}-\lambda_{2}}$ can be obtained approximately by Eq. 5 .

$$
M_{\lambda_{1}-\lambda_{2}}=\frac{C_{1}}{C_{2} / T} \cdot e^{-\frac{c_{2} \cdot X}{T}}\left\{X^{3}+\frac{2}{c_{2} / T} \cdot\left[X^{2}+\frac{2}{c_{2} / T} \cdot\left(\mathrm{X}+\frac{1}{c_{2} / T}\right)\right]\right\}_{X=\frac{1}{\lambda_{1}}}^{X=\frac{1}{\lambda_{2}}}
$$

Because the plane's radiance is mainly decided by the engine and the skin, when calculating the plane radiance, its calculation is divided into three parts: the exhaust, the tail flame, and skin.

The exhaust radiation calculation. In the engineering calculation, gray body radiation model is often used. The cavity made up of the engine is regarded as gray body which emissivity is 0.9. Assume that temperature of all points inside cavity is uniform, and take on the characteristics of the diffuse reflection. Take the exhaust temperature and radiation wavelength range into (4) and (5), and then obtain the exhaust radiance.

The tail flame radiation calculation. According to the fluid mechanics, the flow of gas in the exhaust can be seen as one dimensional steady isentropic flow. After gas passes through the turbine, the static temperature is $T_{1}$ and the static pressure is $P_{1}$. After nozzle the static temperature of the gas is $T_{2}$ and static pressure is $P_{2} . T_{2}$ can be calculated by Eq. 6 .

$$
T_{2}=T_{1}\left(\frac{P_{1}}{P_{2}}\right)^{\frac{r-1}{r}}
$$

$r$ is the heat capacity of gas and it often takes 1.3 for aircraft engine. $P_{2} / P_{1}=0.5$ for turbo jet aircraft engine generally, and thus $T_{2}=0.85 T_{1} \cdot P_{2} / P_{1}=0.4$ for turbofan engine generally, and thus $T_{2}=0.81 T_{1}$. Take tail flame temperature and emissivity into Eq. 4 and Eq. 5, we will obtain the tail flame radiance.

Skin radiation calculation. Skin is an important aspect of affecting plane's infrared radiation because of its large radiation area. The aerodynamic heating make the aircraft's skin temperature undergo dramatic changes, which produce considerable infrared radiation. Radiation of skin can be 
obtained by the method of stagnation temperature. Skin stagnation temperature $T_{s}$ can be expressed as Eq. 7.

$$
T_{s}=T_{0}\left[1+r \cdot \frac{\gamma-1}{2} \cdot M^{2}\right]
$$

The highest temperature aircraft skin could reach depends on the atmospheric temperature, the flight velocity and altitude. At an altitude of $1100 \mathrm{~m}$, skin temperature $T_{s}$ caused by aerodynamic heating can be calculated by empirical formula as Eq. 8 .

$$
T_{s}=T_{0}\left(1+0.164 M^{2}\right)
$$

In the Eq. 7 and Eq. 8, $T_{0}$ is the surrounding atmosphere temperature. $r$ is the temperature recovery coefficient, which is 0.82 for laminar flow, 0.87 for turbulent flow. $\gamma$ is the ratio of air constant pressure heat to constant volume heat, and generally takes 1.4. $M$ is the plane's flight Mach number, that is the ratio of the flight speed to the speed of sound.

When the detector is in different position of the aircraft, the radiance is different.

As shown in the Figure 1, the surrounding space of the aircraft can be divided into three parts. The target radiance of each part can be calculated by Eq. 9, Eq. 10, and Eq. 11.

Part One: $\quad L_{1}=L_{\text {Skin }}$

Part Two: $\quad L_{2}=L_{\text {Skin }}+L_{\text {Exhaust }}+L_{\text {Tailflame }}$

Part Three: $L_{3}=L_{\text {Skin }}+0.5\left(L_{\text {Exhaust }}+L_{\text {Tailflame }}\right)$

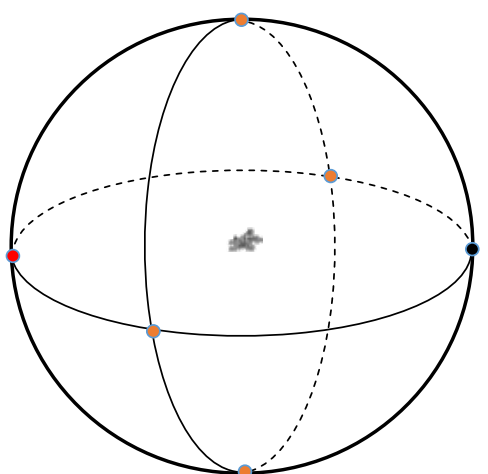

Stereogram

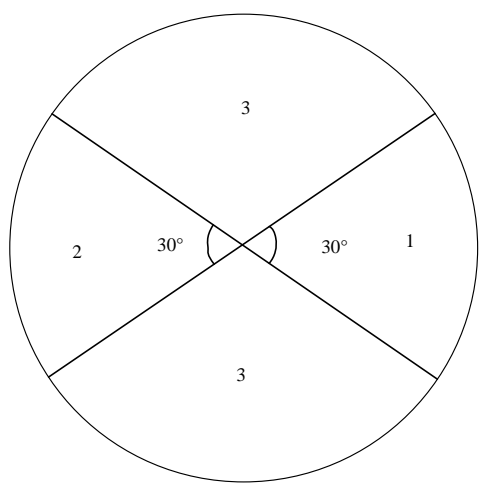

Top View

Figure 1 The relative position of plane and detector

Background radiance model. The main advantages of LOWTRAN7 are quick computation and flexible structure. It is a kind of low resolution software for calculating atmospheric transmittance, and it can meet the requirements of the general infrared system. This paper used LOWTRAN7 to get atmospheric transmittance, solar radiance and atmospheric radiance. In the wavelength $1 \sim 14 \mu \mathrm{m}$, the atmospheric transmittance is 0.529 . The solar radiance is $3209.94 \mathrm{~W} \cdot \mathrm{cm}^{-2} \cdot \mathrm{sr}^{-1}$. The atmospheric radiance is $998.42 \mathrm{~W} \cdot \mathrm{cm}^{-2} \cdot \mathrm{sr}^{-1}$.

Detection probability calculation. The probability of the detector's input SNR exceeding the threshold SNR is the probability of detection. The probability of detection can be expressed as Eq. 12 .

$$
P_{d}\left[S_{N R}\left(\mathrm{~L}_{b}\right)\right]=\frac{1}{\sqrt{2 \pi}} \int_{-\infty}^{S_{N R}\left(\mathrm{~L}_{b}\right)-\mathrm{T}_{N R}} e^{-\frac{\rho^{2}}{2}} d \rho
$$

$S_{N R}=V_{s} / V_{n}$ is input SNR of the detector. $T_{N R}=V-V_{a} / V_{n}$ is threshold SNR of the detector. $\rho=V-V_{a}-V_{s} / V_{n}$ is the variable of integration.

The target detection probability is calculated by Microsoft Visual $\mathrm{C}++6.0$. Program flow chart is shown in figure 2. This program includes one main function and two child functions, which are the radiance function and integral function respectively. Radiance function is used to calculate the exhaust, tail flame and skin radiance in the case of a known temperature and radiation wavelengths. 
Integral function is used to calculate the detection probability. If detection probability is greater than or equal to 0.5 , this paper assumed the detector can detect the plane. If not, it assumed the detector can't detect the plane.

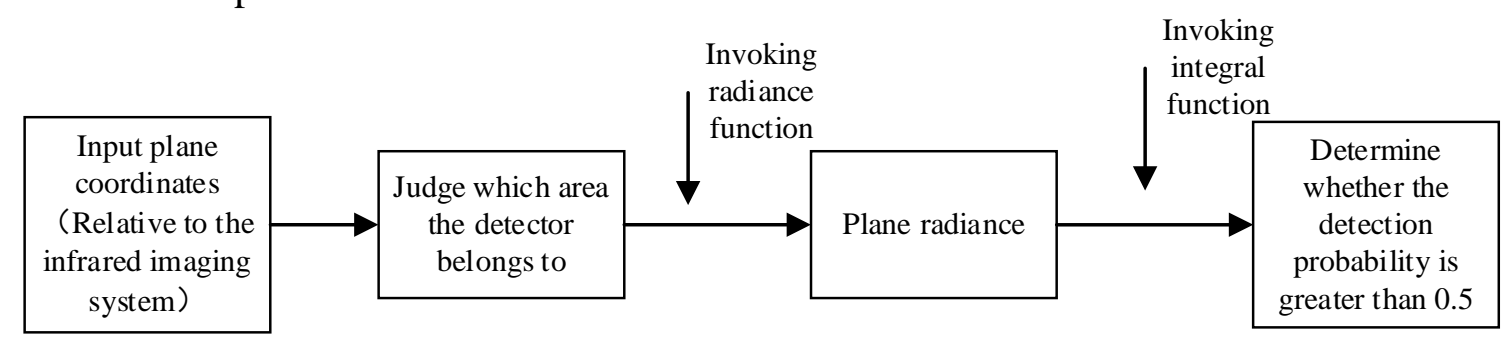

Figure 2 Program flow chart

\section{Conclusions}

In the case of equal distance, detection probability increases as the radiance increases. In the same spherical surface, the impact of the target infrared signal on the detection probability is shown at different orientations.

In part 1, the detector can be detected as far as $816 \mathrm{~m}$. In part 2, as far as $17100 \mathrm{~m}$.In part 3, as far as $11900 \mathrm{~m}$.

In different directions, infrared signal of the target will directly affect the detection probability of infrared detector. Infrared signal in part 1 is the weakest, and detection probability should be minimal in the same distance. Program results also confirm this.

\section{References}

[1] Mao Xia, Chang Le, Diao Weihe. Estimation for detection probability of infrared point target under complex background. Journal of Beijing University of Aeronautics and Astronautics, 2011,37 (11) : 1429-1434.

[2] Xu Meng, Feng Qi, Wei Jun. The Calculation of SNR in SWIR Detector System for Applications to Remote Sensing. Infrared Technology, 2006,28 (10) : 588-590.

[3] Meng Fanbin, Zheng Li. LOWTRAN 7-Based Calculation Method of IR Transmittance in the Atmosphere. Electro-optic Technology Application, 2009,24 (3): 29-32.

[4] Zeng Chong. The modeling and simulation of infrared characteristic of space target. Wuhan, Hubei, China: Huazhong University of Science and Technology.

[5] Wang Mingming, Hao Yingming and Zhu Feng. Modeling and real-time simulation of infrared characteristic of space target. 2012,41 (8) : 1979-1984.

[6] Tian Yupeng. Infrared detection and diagnosis technology. Beijing: Chemical Industry Press. 2006. 5-49.

[7] Ye Yutang, Liu Shuang. Infrared and Low Light Level Technology. Beijing: National Defence Industry Press. 2010. 19-29. 